\title{
CORRECTIONS
}

\section{Publisher Correction: The evolution of multiferroics}

\section{Manfred Fiebig, Thomas Lottermoser, Dennis Meier and Morgan Trassin}

Nature Reviews Materials (2016) https://doi.org/10.1038/natrevmats.2016.46

Published online 05 July 2016

In the key for Figure 1, the legend for the upper right element in the key should read $R^{3+}$, where $R=\mathrm{Sc}, \mathrm{Y}, \operatorname{In}$ or Dy-Lu.

The caption for Figure $1 \mathrm{~b}$ should read: geometrically driven ferroelectricity in hexagonal (h-) $\mathrm{RMnO}_{3}$ emerges from a tilt and deformation of $\mathrm{MnO}_{5}$ bipyramids, which displace the $R$ ions as indicated by the arrows.

The material mentioned in the caption for Figure $1 \mathrm{~d}$ should read (o-) $\mathrm{TbMnO}_{3}$.

In the caption for Figure 2, the reference for the transition from a spiral order to a collinear antiferromagnetic order in orthorhombic $\mathrm{TbMnO}_{3}$ under pressure should be reference 42 .

On page 6, Figure 3a should be called out in the second paragraph, after "establishing a rigid coupling between the antiferromagnetic order parameter of the multiferroic constituent".

On page 8 , in the second paragraph of the "Non-equilibrium dynamics" section the composition for the material should read $\mathrm{LuFe}_{2} \mathrm{O}_{4}$.

On page 9, right column, the symmetry group for $\mathrm{h}-\mathrm{RMnO}_{3}$ should read $\mathrm{P}_{3} \mathrm{c}^{\prime} \mathrm{m}^{\prime}$.

On page 10, in the section "Electromagnons" the second figure callout should be to Figure 1d.

In the caption for Figure 6, the sentence before last should read: the excitations in parts b-d represent electromagnons.

In the section "Nonreciprocal directional dichroism" the reference for unidirectional propagation in $\mathrm{CuB}_{2} \mathrm{O}_{4}$ in magnetic fields should be Ref. 141, whereas the reference for nonreciprocal directional dichroism in gratings should be Ref. 135.

https://doi.org/10.1038/s41578-019-0081-8 I Published online 17 January 2019

\section{Corrected figure}

a Lone pair mechanism

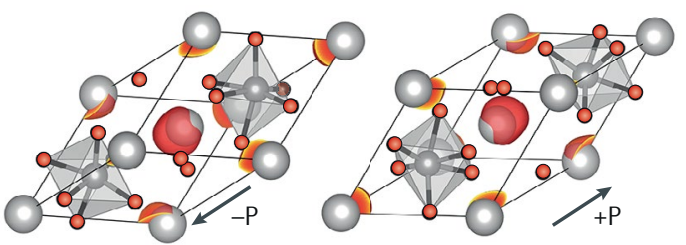

b Geometric ferroelectricity
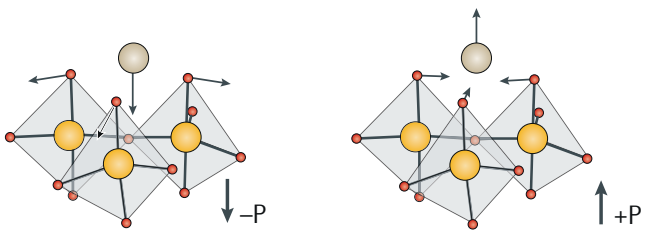

c Charge ordering
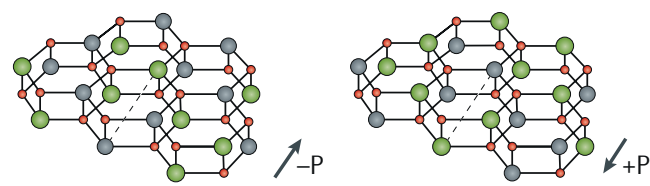

\section{d Spin-driven mechanisms}

Inverse Dzyaloshinskii-Moriya interaction
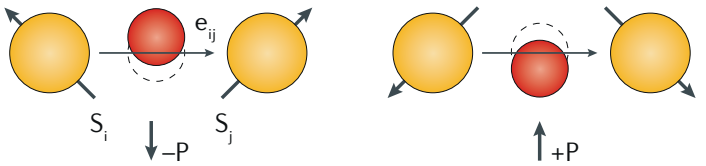

Exchange striction

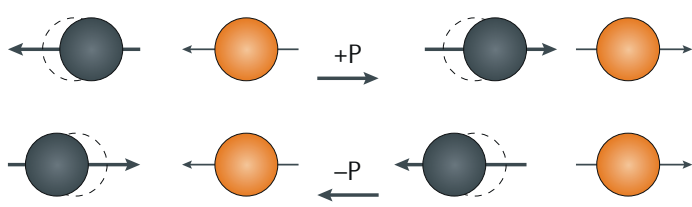

Spin-dependent p-d hybridization
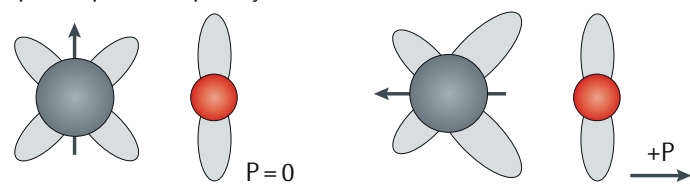

\begin{tabular}{|llll}
$\mathrm{O}^{2-}$ & $\mathrm{Fe}^{2+}$ & $\mathrm{Co}^{2+}$ & $\mathrm{R}^{3+}$ \\
$\mathrm{Bi}^{3+}$ & $\mathrm{Fe}^{3+}$ & $\mathrm{Mn}^{4+}$ & $\mathrm{Mn}^{3+}$
\end{tabular}

Fig. 1 